\title{
Penumbral freeze: travel distance and delays provide an opportunity to study prerecanalization therapy neuroprotection
}

\author{
David J Blacker ${ }^{*} 1$ \\ 'Department of Neurology, Charles Gairdner Hospital \& The Perron Institute for neurological \& translational science, Nedlands, \\ Australia \\ * Author for correspondence: davidblackermd@hotmail.com
}

“Excitingly, stroke patients who had previously been the most disadvantaged could be the first to benefit from new advances in the field of neuroprotection."

First draft submitted: 3 September 2017; Accepted for publication: 28 September 2017; Published online: 10 November 2017

Keywords: distance $\bullet$ ischemic stroke $\bullet$ neuroprotection $\bullet$ penumbral freeze $\bullet$ prerecanalization therapy

The treatment of acute ischemic stroke is currently undergoing a second paradigm shift, to follow the introduction of thrombolytic therapy more than 20 years ago. The recently proven benefits of mechanical thrombectomy [1] is driving change in stroke systems of care [2]. Much of the focus has been on recognition of patients with large vessel occlusion who may potentially benefit from this therapy, and the organization of pathways to enable prompt transportation to appropriate facilities to perform the procedure as quickly as possible. Preliminary results from the DAWN [3] and DEFUSE-3 [4] studies suggest that carefully selected patients with significant penumbra may benefit from revascularization in an extended time window, possibly up to $24 \mathrm{~h}$ after stroke onset. While this suggests that the 'tissue clock' ischemic stroke paradigm is starting to assume more importance, it is likely that the 'time is brain' mantra will always be critical, as suggested by a meta-analysis of the pivotal thrombectomy trials data [5] demonstrating time dependent outcome results. However, if effective strategies to limit infarct growth; in other words, 'penumbral freeze', could be developed, time could potentially become less important, and the window for thrombectomy could be prolonged. Such strategies could be applied at several phases of the 'stroke journey': in the ambulance before arrival at the hospital; soon after arrival at the hospital before thrombectomy; or during an interhospital transfer for patients presenting to centers geographically distant from comprehensive stroke centers (CSCs) where thrombectomy can be performed. This article focuses on this latter group of patients, who are currently disadvantaged because of inevitable delays to receiving the current most effective treatment for ischemic stroke: mechanical thrombectomy. It is possible that improving access to what is increasingly regarded as a standard therapy for stroke, could be coupled with studies of neuroprotective strategies.

The development of a successful neuroprotective agent to improve outcome after ischemic stroke has been a holy grail of neuroscience research [6]. Virtually all agents have failed to make the translational shift from animal models into human clinical practice. One of the reasons is likely to be the delay in administration of the agent. In recent years, the concept of prehospital stroke therapy and research has emerged, in concert with improved systems of stroke care, emerging technologies and the time is brain concept. This provides an opportunity to study administration of neuroprotective agents much sooner after stroke onset. A series of prehospital stroke trials have used paramedics to deliver treatments in the ambulance en route to the hospital. These have included strategies to modulate physiological parameters such as blood glucose [7] and blood pressure [8,9] as well as the early delivery of neuroprotective agents. The largest study to date, the FAST-MAG [10] study, has set the standard for future 'in field' stroke studies. While FAST-MAG did not achieve a positive result, it tackled important practical issues including obtaining consent in the field, randomization and rapid delivery of investigational agents [11]. 
As well as these logistical issues, there are other factors that need consideration in trials of prerecanalization neuroprotective therapies that are likely to impact on the results. Along with the intrinsic effectiveness of the agent, these issues include:

- The relatively weak potency of neuroprotective agents compared with recanalization therapies. Even if an agent is effective, the substantial positive impact of early recanalization may make it very difficult to demonstrate a statistically significant benefit.

- The timing and dosage of neuroprotective agents prior to mechanical thrombectomy. In a metropolitan setting with relatively short transfer times, there may be limited time in which to infuse an optimal dose of agent prior to recanalization.

- The perfusion status of brain tissue (i.e., penumbra) before and during neuroprotective administration, then before and during recanalization, may not be known in patients retrieved, studied and treated in a short time window in metropolitan-based paramedic neuroprotective trials.

An opportunity exists to study some of these issues, while attempting to improve the care for a group of disadvantaged stroke patients: those who live long distances (sometimes several thousands of kilometers) from facilities where stroke intervention is available, such as rural Australia [12], Canada and other geographically large countries. Presently, patients may be transferred centrally over long distances to receive stroke unit care or to access acute stroke interventions if the transfer can be achieved rapidly, within a short time window. The paradigm of 'drip and ship', in other words, the administration of intravenous thrombolysis for ischemic stroke at a distant hospital before transfer to a CSC, has been clinical practice for some years now. This has evolved into a model where 'drip and ship' can also include 'rescue thrombectomy' following arrival at the CSC. In smaller countries, direct transport to CSCs is likely preferred but when thousands of kilometers are involved, this is not an option.

A well established model is now well developed in several regions and is exemplified by the Victorian Stroke Telehealth Network $[13,14]$ in south eastern Australia. Dozens of smaller rural stroke centers are linked to a leading metropolitan CSC with a telehealth service providing rapid access to stroke specialists who assess cases and provide the local medical staff with support for decisions on thrombolysis and transfer to a CSC.

When the full results of the extended time window thrombectomy studies [3,4] are published, there will be intense interest in how to apply this information to stroke patients in rural settings, potentially increasing the likelihood of successful outcome following a long distance interhospital transfer for mechanical thrombectomy. It seems likely that penumbral imaging will be a valuable tool for selecting patients to transfer. For patients requiring a long time window transfer (arbitrarily more than 3-4 h), or possibly any time that takes the patient beyond the upper limit of benefit $(7.3 \mathrm{~h})$ in the meta-analysis dataset [5], clinicians might elect to repeat penumbral imaging when the patient arrives at the CSC in order to have 'fresh' information on which to make a clinical decision. From a research perspective, comparison of the two scans could provide further valuable information on the evolution of infarct and penumbra, and also become a surrogate end point for prerecanalization neuroprotective studies.

With appropriate support and infrastructure, rural sites have the potential to become important study sites for Phase II and III clinical trials of prerecanalization neuroprotective agents. Clinicians would require training in important aspects of clinical trial medicine, but this could be incorporated into the 'activation' of sites, led by experienced trialists who participate in the telehealth component of the service. There would also need to be standardization of neuroimaging techniques across sites.

The pathway for a study subject could be as described below:

- Suspected stroke identified and subject transported to regional site by established clinical pathways;

- Ischemic stroke confirmed by clinical and radiological assessment (including penumbral assessment by computed tomography [CT] perfusion);

- Telehealth consultation and treatment decision by established pathways;

- Telehealth consent for participation in neuroprotective trial;

- Regional site commences treatment with investigational product;

- Air transport to metropolitan center as per established pathways;

- Repeat CT perfusion as a trial end point and component of usual care;

- Mechanical thrombectomy as per usual care;

- Poststroke usual stroke unit care; 
- Clinical trial outcome measures as per typical stroke clinical trial.

This pathway enables a clinical trial to be undertaken alongside routine clinical care. The advantages over a metropolitan-based system include the potential to: administer an optimal dose of investigational agent prior to thrombectomy; study a surrogate end point as a component of routine care, in other words, repeat CT perfusion; and, further enhance the clinical pathway and care for stroke patients who previously had limited access to interventional stroke services.

Some promising agents include the NA-1 compound that is currently in early-phase human studies [15] and arginine-rich peptides [16] that seem comparatively more effective in animal studies. Ongoing carefully designed animal studies could actually be modeled on the new human stroke paradigm of rapid recanalization, and seek to explore how long the time window for thrombectomy might be prolonged.

Regions with geographically dispersed or isolated populations such as Australia, Canada and parts of the USA could potentially develop this research paradigm alongside routine clinical care, and with collaboration lead to rapid gains in knowledge. Excitingly, stroke patients who had previously been the most disadvantaged could be the first to benefit from new advances in the field of neuroprotection.

\section{Financial \& competing interests disclosure}

The author has no relevant affiliations or financial involvement with any organization or entity with a financial interest in or financial conflict with the subject matter or materials discussed in the manuscript. This includes employment, consultancies, honoraria, stock ownership or options, expert testimony, grants or patents received or pending, or royalties.

No writing assistance was utilized in the production of this manuscript.

\section{References}

1 Badhiwala JH, Nassiri F, Alhazzani W et al. Endovascular thrombectomy for acute ischemic stroke: a meta-analysis. JAMA 314(17), 1832-1843 (2015).

2 Mocco J, Fargen KM, Goyal M et al. Neurothrombectomy trial results: stroke systems, not just devices, make the difference. Int. J. Stroke 10(7), 990-993 (2015).

3 Jovin TG, Saver JL, Ribo M et al. Diffusion-weighted imaging or computerized tomography perfusion assessment with clinical mismatch in the triage of wake up and late presenting strokes undergoing neurointervention with Trevo (DAWN) trial methods. Int. J. Stroke 12(6), 641-652 (2017)

4 Albers GW, Lansberg MG, Kemp S et al. A multicenter randomized controlled trial of endovascular therapy following imaging evaluation for ischemic stroke (DEFUSE 3). Int. J. Stroke 12(8), 896-905 (2017).

5 Goyal M, Menon BK, van Zwam WH et al. Endovascular thrombectomy after large-vessel ischaemic stroke: a meta-analysis of individual patient data from five randomised trials. Lancet 387(10029), 1723-1731 (2016).

6 Fisher M, Ratan R. New perspectives on developing acute stroke therapy. Ann. Neurol. 53(1), 10-20 (2003).

7 Nurmi J, Lindsberg PJ, Häppölä O, Klemetti E, Westerbacka J, Castren M. Strict glucose control after acute stroke can be provided in the prehospital setting. Acad. Emerg. Med. 18(4), 436-439 (2011).

8 Shaw L, Price C, McLure S et al. Paramedic Initiated Lisinopril For Acute Stroke Treatment (PIL-FAST): study protocol for a pilot randomised controlled trial. Trials 12, 152 (2011).

9 Ankolekar S, Sare G, Geehange C et al. Determining the feasibility of ambulance-based randomised controlled trials in patients with ultra-acute stroke: study protocol for the 'Rapid Intervention with GTN in Hypertensive Stroke Trial' (RIGHT). Stroke Res. Treat. 2012, 385753 (2012).

10 Saver JL, Starkman S, Eckstein M et al. Prehospital use of magnesium sulfate as neuroprotection in acute stroke. N. Engl. J. Med. 372, 528-536 (2015).

11 Hatez $S$. The beneficial failure of the FAST-MAG trial. Prehospital magnesium didn't help stroke patients, but we still learned a lot. EMS World 43(7), 58-60 (2014).

12 Blacker DJ, Hankey GJ. Prehospital stroke management: an Australian perspective. Intern Med. J. 44(12a), 1151-1153 (2014).

13 Cadilhac DA, Moloczij N, Denisenko S et al. Establishment of an effective acute stroke telemedicine program for Australia: protocol for the Victorian Stroke Telemedicine project. Int. J. Stroke 9(2), 252-8 (2014).

14 Davis SM, Campbell BC, Donnan GA. Endovascular thrombectomy and stroke physicians: equity, access and standards. Stroke 48(8), 2042-2044 (2017).

15 Hil MD, Martin RH, Mikulis D et al. Safety and efficacy of NA-1 in patients with iatrogenic stroke after endovascular aneurysm repair (ENACT): a Phase 2, randomised, double-blind, placebo-controlled trial. Lancet Neurol. 11(11), 942-950 (2012). 
16 Meloni BP, Milani D, Cross JL et al. Assessment of the neuroprotective effects of arginine-rich protamine peptides, poly-arginine peptides (R12-Cyclic, R22) and arginine-tryptophan-containing peptides following in vitro excitotoxicity and/or permanent middle cerebral artery occlusion in rats. Neuromolecular Med. doi: 10.1007/s12017-017-8441-2 (2017) (Epub ahead of print). 